\title{
Long-term comparative and prospective cohort study of renal function in patients with HIV infection treated with tenofovir disoproxil fumarate
}

\author{
Guillermo Maestro de la Calle ${ }^{1}$, Federico Pulido ${ }^{1}$, Matilde Sánchez-Conde², \\ Juan Carlos López Bernaldo de Quirós ${ }^{3}$ \\ ${ }^{1}$ Department of HIV Infection, 12 de Octubre University Hospital, Madrid, Spain \\ ${ }^{2}$ Internal Medicine Service, Ramón y Cajal University Hospital, Madrid, Spain \\ ${ }^{3}$ Department of HIV Infection, Gregorio Marañón University Hospital, Madrid, Spain
}

\begin{abstract}
Introduction: Evidence regarding long-term evolution of renal function in patients with human immunodeficiency virus (HIV) infection treated with tenofovir disoproxil fumarate (TDF) is scarce and often retrospective.

Material and methods: We carried out an observational prospective cohort study. Patients with serum creatinine lower than $1.2 \mathrm{mg} / \mathrm{dl}$ and an estimated glomerular filtration rate (eGFR) higher than $60 \mathrm{ml} / \mathrm{min} / 1.73 \mathrm{~m}^{2}$ were included between January 2001 and December 2005. The primary outcome was the onset of a clinically relevant decrease in renal function (CRDRF) defined as an eGFR $<60 \mathrm{ml} / \mathrm{min} / 1.73 \mathrm{~m}^{2}$ in two consecutive measures or $<50 \mathrm{ml} / \mathrm{min} / 1.73 \mathrm{~m}^{2}$ in any measure. Secondary objectives were to identify risk factors for the emergence of CRDRF and renal recovery after TDF discontinuation.

Results: Seventy patients receiving TDF and 58 controls were included. After a median follow-up of 7.6 years, 10 patients in the TDF group and none in the control group developed CRDRF $(p=0.005)$. The incidence rate of CRDRF was 2.2 cases per 100 treated patients per year (CI 95\%: 0.85-3.62). Risk factors for CRDRF onset were higher baseline age (HR = 1.1, CI 95\%: 1.05-1.18; $p=0.001$ ), arterial hypertension $(\mathrm{HR}=24.8$, CI 95\%: 3.3-185.6; $p=0.002)$ and lower baseline eGFR $(\mathrm{HR}=0.93$, CI 95\%: 0.89-0.97; $p=0.002$ ). After 36 months from TDF discontinuation in CRDRF cases, seven patients achieved renal recovery (eGFR $>60 \mathrm{ml} / \mathrm{min} / 1.73 \mathrm{~m}^{2}$ in $\geq 2$ consecutive measures, with six months between each measure).
\end{abstract}

Conclusions: Long-term use of TDF in the treatment of HIV infection is associated with a higher incidence of a clinically relevant decrease in renal function which may be only partially reversible.

Key words: HIV, tenofovir disoproxil fumarate, kidney disease, long-term effects.

Address for correspondence: Dr. Guillermo Maestro de la Calle, Department of HIV Infection, 12 de Octubre University Hospital, Avenida de Córdoba S/N, 28041 Madrid, Spain, phone: +34620823653, e-mail: gmaestro@gmail.com
Article history:

Received: 23.10.2016

Received in revised form: 15.11.2016

Accepted: 03.01.2017

Available online: 29.03 .2017
International Journal of HIV-Related Problems

HIV \& AIDS

R e v i e w 


\section{Introduction}

The risk of both acute kidney injury and chronic renal disease is increased in human immunodeficiency virus (HIV) patients compared with the general population [1]. After the introduction of highly active antiretroviral treatment (HAART), arterial hypertension, diabetes, atherosclerosis and drug toxicity are gaining relevance in the HIV patient as risk factors for the development of kidney disease.

Since tenofovir disoproxil fumarate (TDF) approval in 2000, there is growing evidence of its relationship with nephrotoxicity. The drug is mainly excreted in the kidney through its filtration in the glomerulus and active tubular secretion. It is postulated that TDF-related kidney damage could be favoured either by means of disruption of the function of tubular proteins (mainly anion transporter protein 1 and drug resistant protein 4$)[2,3]$ or as a result of mitochondrial toxicity secondary to intracellular accumulation of the molecule [4]. Kidney damage with tubular dysfunction has been related with TDF use, even without a decrease in the estimated glomerular filtration rate [5].

In a large meta-analysis including clinical trials and cohort studies addressed to study renal safety of TDF, only a slight significant difference was observed in patients treated with TDF as compared with other ART regimens or placebo $(-3.90 \mathrm{ml} / \mathrm{min}$; CI $95 \%[-5.66,-2.14])$ after a median follow-up of 60 weeks [6]. Moreover, large clinical trials with long follow-up periods of more than 144 weeks in naïve patients have shown a renal safety profile of ART containing TDF, either similar to other ART without it, or with small significant declines in the estimated glomerular filtration rate (eGFR) of clinical implications difficult to ascertain [79]. Observational studies with longer follow-up periods suggest a more deleterious effect on the renal function, and point that TDF is associated with a higher incidence of chronic kidney disease [10-17]. Overall, these results have shown either a modest or absent decline in renal function associated with TDF use after short follow-up periods of months or very few years, while studies with longer follow-up periods suggest a more relevant decrease in renal function [13-17].

The primary objective of this study was to assess the incidence of a clinically relevant decrease in renal function (CRDRF) in patients treated with TDF as compared with controls. Secondary objectives were the evaluation of risk factors for the development of CRDRF and the reversibility of eGFR decline after cessation of TDF in patients that experienced CRDRF.

\section{Material and methods}

\section{Study design and setting}

We conducted a prospective cohort study in which patients were recruited between January 2002 and December 2005 from Gregorio Marañón Hospital, a tertiary care university hospital located in Madrid (Spain) in which about 2,500 patients with HIV infection are followed in 10 different consultations. Patient recruitment was made in one of these ten consultations. Data collection and patient follow-up was finished in June 2013, with a median follow-up of 7.6 years (IQR 4.1 to 9.0) after baseline visit.

\section{Participants}

Seventy patients initiated TDF as part of their ART, all of which were offered to collaborate and gave oral consent to participate in the study. Fifty-eight controls initiating a nonTDF containing regimen were recruited and matched with the TDF group for age, sex, serum creatinine, height and weight. Antiretroviral treatment regimens were composed according to the Spanish ART guidelines during the recruiting period [18]. Inclusion criteria for participating and data analysis were age $>18$ years, baseline serum creatinine $<1.2 \mathrm{mg} / \mathrm{dl}$ and a eGFR $>60 \mathrm{ml} / \mathrm{min} / 1.73 \mathrm{~m}^{2}($ MDRD-4) and at least two follow-up visits with plasma HIV load $<50$ copies $/ \mathrm{ml}$ (whenever this threshold was achieved). Patients were followed in the ambulatory setting according to an established schedule of visits to the HIV hospital unit.

\section{Variables and other data measurements}

The main outcome was the CRDRF related with TDF use, and was defined as the presence of eGFR $<60 \mathrm{ml} / \mathrm{min} / 1.73 \mathrm{~m}^{2}$ in two consecutive measures with at least 3 months between each measure, or $<50 \mathrm{ml} / \mathrm{min} / 1.73 \mathrm{~m}^{2}$ (MDRD-4) in any measure, when no other attributed cause was evident. The former eGFR threshold was used since it is correlated with higher morbidity and mortality [19], and the latter because of clinical concern about a potential abrupt eGFR decline. Secondary outcomes were the identification of risk factors for the development of CRDRF, the reversibility of CRDRF related to TDF use, and analysis of the diagnostic performance of renal parameters as diagnostic tools for early identification of CRDRF. Renal recovery after TDF discontinuation was defined as eGFR $>60 \mathrm{ml} / \mathrm{min} / 1.73 \mathrm{~m}^{2}$ in 2 or more consecutive measures, with six months between each measure.

For secondary outcomes (e.g. eGFR recovery), the Chronic Kidney Disease Epidemiology Collaboration equation (CKD-EPI) was used to estimate the GFR as these outcomes were retrospectively analysed and this equation has more recently become the standard measurement for renal function in subjects with eGFR $>60 \mathrm{ml} / \mathrm{min} / 1.73 \mathrm{~m}^{2}$ [20] and has been validated in the HIV population [21]. Serum creatinine measure was performed with standardized tests (Creatinine Jaffé Gen 2, Roche) in every visit, and so height and weight were measured, in order to identify potential confounders of serum creatinine levels. Calculation of serum creatinine and eGFR (CKD-EPI) variation over time was performed subtracting their baseline data from follow-up measures. Frequency of data registration was at month $3,6,912$, then every 6 months until month 72, and then every 12 months.

Other data such as age, sex, arterial hypertension, diabetes, dyslipidemia, tobacco use, HCV and HBV status (positive serol- 
ogy for $\mathrm{HCV}$, and positive HBs-antigen for HBV), date of HIV diagnosis, way of HIV transmission, plasma nadir HIV load and CD4+ lymphocytes, previous AIDS-defining events, and ART components, and potentially nephrotoxic drugs were registered.

\section{Statistical analysis}

Quantitative variables were described with mean or median with $95 \%$ confidence interval (95\% CI) or interquartile range (IQR). When comparing quantitative variables, Student's $t$-test or Mann Whitney $U$-test were used according to the normal or non-normal distribution of the variables. Qualitative variables were described by their percentage, and compared with $\mathrm{X}^{2}$ test or Fisher's exact test when necessary. Differences between groups in terms of the survival curves of CRDRF were analysed with the Kaplan-Meier method, and log-rank test. Univariate Cox regression was used to identify risk factors for the development of CRDRF, and haz- ard ratios (HR) were subsequently adjusted in a multivariate model by stepwise backward regression with variables that proved to be significant in the univariate model. We established a cut-off $p$-value for Type I error of 0.05 to state significant differences. Data were analysed with IBM SPSS statistics software version 20 for Mac Os X.

Diagnostic performance of renal function parameters to predict the emergence of CRDRF was studied with ROC curves. Creatinine levels at months 3,12 and 30 were chosen since no CRDRF events were registered at these time points. In month 12 and 30, one and two patients, respectively, had already developed CRDRF, and were managed by using their penultimate serum creatinine for estimating ROC curves in these months.

\section{Results}

Of the 128 patients involved in the study, 70 received TDF as part of their ART regimen (TDF group) and 58 re-

Table 1. Baseline characteristics*

\begin{tabular}{|c|c|c|}
\hline Factor & Control group $(n=58)$ & TDF group $(n=70)$ \\
\hline Age (years) & $40.9(36.4-47.1)$ & $39.9(36.7-44.5)$ \\
\hline Males & $43(74.1)$ & $56(80.0)$ \\
\hline Hypertension & $3(5.2)$ & $6(8.6)$ \\
\hline Diabetes & $9(15.5)$ & $11(15.7)$ \\
\hline Current smoker & $18(31.0)$ & $31(44.3)$ \\
\hline Dyslipidemia & $2(3.4)$ & $5(7.1)$ \\
\hline Weight (kg) & $69.0(59.0-78.0)$ & $65.0(58.2-72.0)$ \\
\hline BMI $\left(\mathrm{kg} / \mathrm{m}^{2}\right)$ & $23.7(21.3-26.0)$ & $23.0(21.1-24.7)$ \\
\hline HCV infection & $18(31.0)$ & $32(45.7)$ \\
\hline $\mathrm{HBV}^{+}$ & $2(3.4)$ & $21(30.0)$ \\
\hline IDU transmission of HIV & $21(36.2)$ & $33(47.1)$ \\
\hline $\mathrm{T}_{\text {infection HIV }}(\text { years })^{\dagger}$ & $5.0(1.7-11.0)$ & $9.8(4.6-13.2)$ \\
\hline Naïve patient ${ }^{\dagger}$ & $19(32.8)$ & $9(12.9)$ \\
\hline $\mathrm{T}_{\text {previous ART }}(\text { months })^{\dagger}$ & $31.2(0.0-71.3)$ & $81.6(26.6-109.9)$ \\
\hline Previous AIDS defining event & $14(24.1)$ & $20(28.6)$ \\
\hline Peripheral blood CD4+ lymphocytes (cells/mL) & $345.1^{\mathrm{M}}(292.1-398.1)$ & $372.1^{M}(316.9-427.2)$ \\
\hline Nadir CD4+ & $187.6^{\mathrm{M}}(154.7-220.4)$ & $182.1^{M}(148.7-215.6)$ \\
\hline CD4+ $<200$ cells $/ \mathrm{ml}$ & $13(22.4)$ & $17(24.3)$ \\
\hline CD4+ $<100$ cells $/ \mathrm{ml}$ & $7(12.1)$ & $4(5.7)$ \\
\hline Plasma HIV viral load (copies/ml) & 5.022 (49. 71.719] & $1.930[49.42 .208]$ \\
\hline$<50$ copies $/ \mathrm{ml}$ & $26(44.8)$ & $28(40.0)$ \\
\hline$\geq 100000$ copies $/ \mathrm{ml}$ & $11(19.0)$ & $11(15.7)$ \\
\hline Creatinine (mg/dl) & $0.9(0.8-1.0)$ & $0.9(0.7-1.0)$ \\
\hline eGFR with MDRD $\left(\mathrm{ml} / \mathrm{min} / 1.73 \mathrm{~m}^{2}\right)$ & $91.1(81.9-104.9)$ & $92.7(82.7-106.3)$ \\
\hline eGFR with CKD-EPI (ml/min/1.73 m²) & $101.7(93.0-109.9)$ & $106.9(92.4-111.7)$ \\
\hline \multicolumn{3}{|c|}{$\begin{array}{l}{ }^{*} \text { Values in square brackets are the } 95 \% \text { confidence interval (or the interquartile range) preceded by the mean (or median). Values in parentheses are the } \\
\text { percentage preceded by the number of patients } \\
M \text { - mean value (normal distribution), }{ }^{+} \text {Significant differences were found with p value }<0.05 \\
B M I \text { - body mass index, HBV - hepatitis B infection, HCV-hepatitis C infection, IDU - intravenous drug user, eGFR-estimated glomerular filtration rate, } \\
T_{\text {infection HIV }} \text { - time of infection of HIV from diagnosis, } T_{\text {TAR previous }} \text { - time of TAR in treatment experienced patients }\end{array}$} \\
\hline
\end{tabular}


Table 2. Baseline antiretroviral regimens*

\begin{tabular}{|c|c|c|}
\hline & Control group $(n=58)$ & TDF group $(n=70)$ \\
\hline NRTI & $68(97.1)$ & $57(98.3)$ \\
\hline †3TC & $54(93.1)$ & $44(62.9)$ \\
\hline $\mathrm{d} 4 \mathrm{~T}$ & $6(10.3)$ & $7(10.0)$ \\
\hline${ }^{\dagger} \mathrm{AZT}$ & $11(19.0)$ & $4(5.7)$ \\
\hline$A B C$ & $11(19.0)$ & $9(12.9)$ \\
\hline${ }^{\dagger} \mathrm{ddl}$ & $38(65.5)$ & $16(22.9)$ \\
\hline $\mathrm{NNRTI}^{\dagger}$ & $43(89.6)$ & $29(41.4)$ \\
\hline${ }^{\dagger} \mathrm{EFV}$ & $19(32.8)$ & $12(17.1)$ \\
\hline NVP & $24(41.4)$ & $17(24.3)$ \\
\hline $\mathrm{Pl}^{+}$ & $14(24.1)$ & $44(62.8)$ \\
\hline${ }^{\dagger} \mathrm{LPV}$ & $10(17.2)$ & $35(50.0)$ \\
\hline ATV & $1(1.7)$ & $3(4.3)$ \\
\hline FPV & $0(0)$ & $4(5.7)$ \\
\hline NFV & $2(3.4)$ & $1(1.4)$ \\
\hline TPV & $0(0)$ & $1(1.4)$ \\
\hline APV & $1(1.7)$ & $0(0)$ \\
\hline T20 & $0(0)$ & $1(0.4)$ \\
\hline
\end{tabular}

*Values in parentheses are the percentage preceded by the number of patients tSignificant differences were found with $p$ value $<0.05$

$\mathrm{NNRTI}$ - non-nucleoside reverse transcriptase inhibitor, $\mathrm{NRTI}$ - nucleoside reverse transcriptase inhibitor (two NRTI as part of the treatment regimen), $\mathrm{Pl}$-protease inhibitor

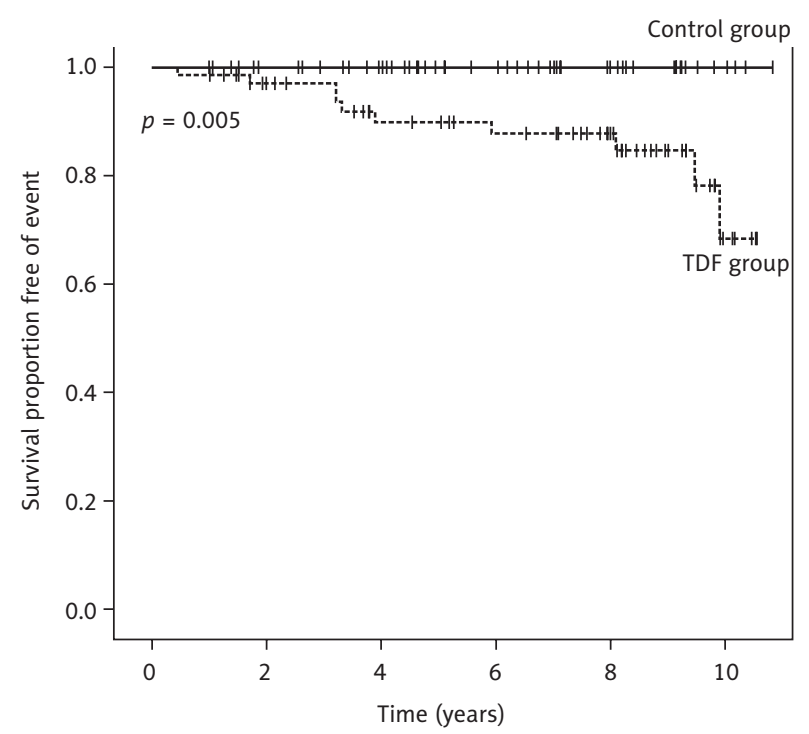

No. at risk

$\begin{array}{rrrrrrr}\text { Control } & 58 & 53 & 44 & 29 & 17 & 4 \\ \text { TDF } & 70 & 59 & 47 & 42 & 29 & 5\end{array}$

Figure 1. Kaplan-Meier curves for clinically relevant decrease in renal function (CRDRF). Cumulative event rates for the CRDRF in tenofovir disoproxil fumarate (TDF) and control groups during the overall study period. Vertical lines show censored patients ceived an ART regimen without it (control group). Every patient was included in the statistical analysis, even those who were lost in follow-up (5\% in the TDF group vs. $10 \%$ in the control group, $p=0.32$ ). There were no significant differences in most of the baseline demographic data (Table 1), but we found significant differences in terms of the presence of chronic HBV infection (30\% TDF group vs. 3.4\% control group, $p=0.0001)$, time from HIV infection diagnosis ( 9.8 years in the TDF group vs. 5.0 years in the control group, $p=0.01$ ), number of ART naive patients (12.9\% TDF group vs. $32.8 \%$ control group, $p=0.007$ ) and time from first ART in previously treated patients (81.6 years in the TDF group vs. 31.2 years in the control group, $p=0.0001$ ). In terms of the ART regimens (Table 2), most patients received two nucleoside analogue reverse-transcriptase inhibitors as the backbone of their ART regime. TDF group patients received more protease inhibitors than the control group (62.8\% vs. $24.1 \%$; $p=0.001)$, mainly due to the use of boosted lopinavir $(50.0 \%$ TDF group vs. $17.2 \%$ control group, $p=0.0001$ ).

After a median follow-up of 7.6 years (IQR 4.1 to 9.0), 10 patients in the TDF group developed CRDRF and none in the control group ( $p=0.005, \log$ rank; Figure 1). There were no significant differences in follow-up times (TDF 7.7 years (IQR 3.3 to 8.9 ) versus control 6.1 years (IQR 4.1 to 8.3), $p=0.44$. The cumulative incidence of CRDRF in the TDF group was 14.3 cases per 100 treated patients, and the incidence rate was 2.2 cases per 100 treated patients per year $(95 \%$ CI: 0.85 to 3.62). Loss of follow-up or death took place in $10.9 \%$ of the patients ( 9 and 5 patients, respectively), without significant differences between groups. There were no significant differences in terms of censored causes between TDF and control groups in terms of mortality ( $6.7 \%$ vs. $1.7 \%$; $p=0.37)$, loss of follow-up (5.0\% vs. $10.3 \%$; $p=0.32)$, and initiation of other nephrotoxic treatments $(3.3 \%$ vs. $1.7 \%$; $p=1.0$ ). Significant differences were identified in patients censored because of changes in the ART regimen (stopping TDF for other reason in the TDF group in $35.0 \%$ vs. initiation of TDF in the control group in $67.2 \%, p=0.001$ ).

Baseline characteristics were studied as potential risk factors for the development of CRDRF in the TDF group using univariate Cox regression model. Higher basal age (HR 1.1 for each year, 95\% CI: 1.05 to $1.18, p=0.001$ ), the presence of arterial hypertension (HR 8.3, 95\% CI: 2.1 to 33.3, $p=0.003)$, lower GFR estimated with CKD-EPI equation (HR 0.93 for each $\mathrm{ml} / \mathrm{min} / 1.73 \mathrm{~m}^{2}$ CKE-EPI, 95\% CI: 0.89 to $0.97, p=0.002)$, treatment with atazanavir $(\mathrm{HR}=7.6$, $95 \% \mathrm{CI}: 1.5-36.9, p=0.01)$ and intravenous drug $(\mathrm{HR}=0.12$, $95 \%$ CI: 0.01 to $0.95, p=0.04$ ) were all identified as significant. In the multivariate model including all significant variables of the univariate model, only age, hypertension and baseline eGFR preserved statistical significance, and only in the case of hypertension the HR was modified ( $\mathrm{HR}=24.8$; 95\% CI: 3.3-185.6, $p=0.002$ ).

The incidence of CRDRF took a bimodal pattern over time, with two peaks of incidence. The first one clustered CRDRF cases in the first 4 years of follow-up, and the second one that showed up after the $8^{\text {th }}$ year until the end of follow-up 


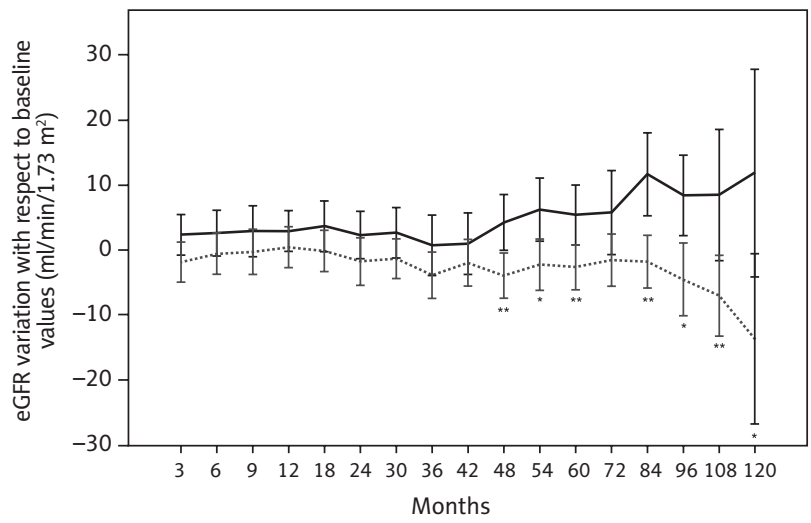

No. Patients

$\begin{array}{rllllllllc}\text { Control } & 58 & 58 & 53 & 49 & 43 & 34 & 25 & 19 & 7 \\ \text { TDF } & 70 & 68 & 62 & 57 & 49 & 47 & 41 & 39 & 13\end{array}$

Figure 2. Variation of the estimated glomerular filtration rate (eGFR) (CKD-EPI equation) with respect to baseline values over time. A dotted line represents the tenofovir disoproxil fumarate (TDF) group (patients who developed DRF excluded), while a solid line represents the control group. A vertical line represents the $95 \%$ confidence intervals around mean values. * indicates that statistically significant differences were found with $p<0.05$ and $^{* *}$ with $p<0.01$

(Figure 1). This suggested the presence of two types of scenarios for the presentation of CRDRF, an early presentation group and a delayed one. In the early presentation group (first 43.2 months), patients had a lower basal renal functional reserve (baseline eGFR with CKD-EPI equation, $80.6 \pm 9.2$ vs. $\left.92.9 \pm 6.3 \mathrm{ml} / \mathrm{min} / 1.73 \mathrm{~m}^{2}, p=0.016\right)$ and tended to be older $(54.5 \pm 21.2$ vs. $44.1 \pm 10$ years, $p=0.27)$, and with a higher prevalence of arterial hypertension ( $40 \%$ vs. $20 \%, p=1.0$ ), than in the delayed presentation group.

The evolution of the eGFR over time was significantly different between groups after the patients who experienced the CRDRF were excluded from the comparison (Figure 2). In month 48, patients in the TDF group (patients who had experienced the CRDRF excluded) experienced a progressive decline in their eGFR as compared with the control group (median variation $0.00 \mathrm{IQR}-10.86$ to 0.00 in the TDF group versus +4.92 IQR -5.08 to $+12.23 \mathrm{ml} / \mathrm{min} / 1.73 \mathrm{~m}^{2}$ in the control group; $p=0.007)$. Thereafter, we found significant differences in eGFR variations between groups in almost every month and these differences became greater over time. As an example, in month 84 , the median eGFR variation was 0.00 $\mathrm{ml} / \mathrm{min} / 1.73 \mathrm{~m}^{2}(\mathrm{IQR}-7.58$ to +5.46$)$ in the TDF group versus $+11.51(\mathrm{IQR}+5.01$ to +17.53$)$ in the control group $(p=0.001)$.

After TDF discontinuation because of CRDRF, we studied the reversibility of renal function. After 36 months of follow-up, seven of these patients achieved a renal recovery. The median time to renal recovery was 18 months. None of the patients returned to his baseline eGFR, and after the end of follow-up the median eGFR was $68.3 \mathrm{ml} / \mathrm{min} / 1.73 \mathrm{~m}^{2}$ (IQR: 60.4 to 79.2 ), compared with their median baseline

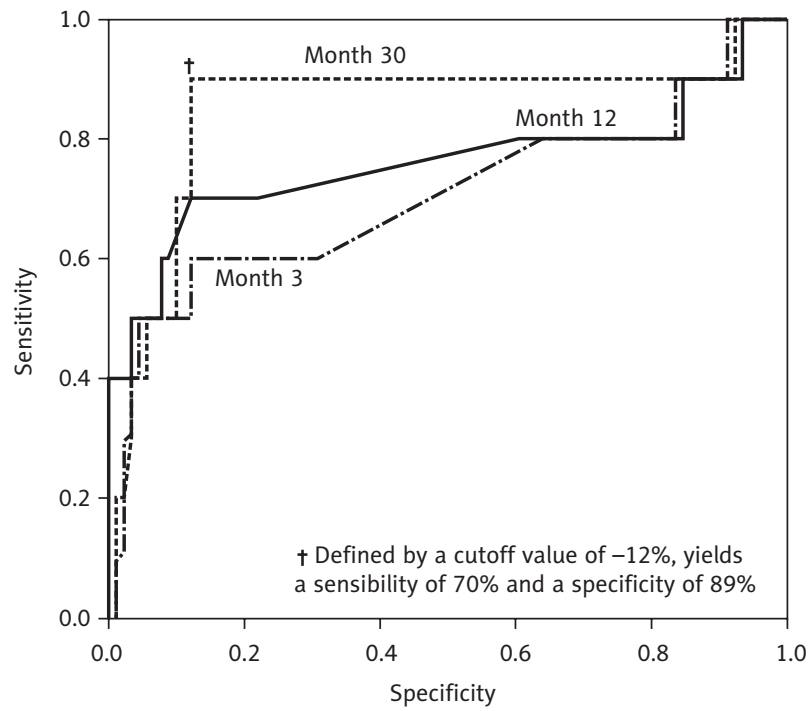

Figure 3. Receiver operating characteristic (ROC) curves using the percent variation of estimated glomerular filtration rate (eGFR) (CKD-EPI equation) with respect to baseline values. Best AUC was found at month 30 (0.85, 95\% Cl: 0.67-1.0)

eGFR of $88.9 \mathrm{ml} / \mathrm{min} / 1.73 \mathrm{~m}^{2}$ (IQR: 83.2 to 97.6 ). Only a higher time of TDF exposure was a predictor of renal recovery in the univariate and multivariate Cox's regression model (HR 1.03 per month of exposure; 95\% CI: 1.002-1.05; $p=0.03$ ).

Finally, we studied the diagnostic performance of the variation of creatinine and the eGFR (as well as their percentage) in months 3, 12 and 30 as early diagnostic tools of CRDRF onset in patients who received TDF as part of their ART. When choosing the cut-off value, a higher specificity value prevailed over higher sensitivity values. We found the percentage of eGFR variation in month 30 to have the best diagnostic performance, with an area under the curve of 0.85 (95\% CI: $0.67-1.0)$, with a cut-off point in $-12 \%$ that offers a sensibility of $70 \%$ and a specificity of $89 \%$ (Figure 3 ).

\section{Discussion}

In this cohort study the use of TDF in the treatment of HIV infection was related with a higher incidence of CRDRF. An older age, arterial hypertension and lower baseline eGFR were significant risk factors for the development of this event. Even when patients who developed CRDRF were excluded from the analysis, TDF group patients experienced significant decreases in their eGFR compared with the control group in almost every month after 4 years of treatment. Following TDF discontinuation, most of the patients achieved an eGFR higher than $60 \mathrm{ml} / \mathrm{min} /$ $1.73 \mathrm{~m}^{2}$ (CKE-EPI).

Overall both groups had similar baseline characteristics. However, an important limitation of the study is a prescription bias by which patients that received TDF had more comorbidities. This phenomenon can be explained by the guideline recommendations during the recruitment period, which 
recommended TDF in the setting of rescue therapy instead of a component of ART for treating naïve patients, which has been recommended in more recent guidelines [18]. In this sense, the TDF group had a significantly higher time from HIV infection diagnosis, fewer ART naïve patients and longer times from first ART, which makes a more fragile population more susceptible to renal disease. Moreover, this bias could explain another significant limitation of our work, which is the higher prevalence of boosted protease inhibitor (PI) as part of the ART regimen in TDF group patients. Boosted PIs have been related to a higher incidence of kidney disease in many observational studies [10, 22], although data from clinical trials suggest a reasonable renal safety profile [23]. In our study, the use of PIs did not show any significant relationship with CRDRF so its role as a potential confounder in our study might be limited.

A remarkable strength of this study is the long follow-up period in comparison with clinical trials and other prospective cohort studies published to date $[6,7,10,24]$. Of interest, the median time to CRDRF event was 3.6 years, which is much longer than most follow-up periods in other studies. This may have made it possible for our study to detect this progressive event despite a limited sample size by identifying late events. It is remarkable that a bimodal history of progression to CRDRF was observed with kidney injury events being clustered in the first four years and after the eighth year of TDF treatment. This is a novel finding and it suggests that patients with more baseline risk factors for kidney injury emergence (e.g., age, hypertension, lower baseline eGFR) may progress to CRDRF in the first years of treatment, while in the healthier counterparts kidney injury may only become apparent after several years of TDF exposure.

While most previously published works with shorter follow-up periods have found little variation of the eGFR related to TDF use [6], some observational studies with longer follow-up periods have found CRDRF related with TDF use [13-17]. It is worth mentioning some considerations about these studies. Some of them have been conducted in specific populations such as African population [16], among patients with low body weight [15], or treatment-naïve patients [17], and others have used the Cockcroft-Gault equation to estimate the creatinine clearance [13], which can result in an overestimate of the creatinine clearance. These situations might explain why our study shows a higher incidence of chronic kidney disease related to TDF use in the long term. Due to our scarce sample size, subtle but significant eGFR variations were only observed after four years of treatment (patients with CRDRF excluded from the analysis), and were similar in their magnitude with previously published observational works $[6,10]$.

Risk factors for TDF related kidney damage are not clearly established. Observational studies suggest that older age and lower baseline eGFR are risk factors, all of which are well-known risk factors for progression to chronic kidney disease in the general population, with arterial hypertension not being so clearly related $[10,12,14]$. We only found these three risk factors as significant predictors of CRDRF onset in the multivariate model. Other more specific factors related in other studies with TDF nephrotoxicity, such as concomitant use of protease inhibitors, were not found to be significant risk factors but sample size limits our ability to draw any conclusions in this regard.

When TDF was discontinued because of CRDRF development most patients achieved a sustained eGFR over $60 \mathrm{ml} / \mathrm{min} / 1.73 \mathrm{~m}^{2}$ (CKD-EPI) after 3 years' follow-up, but none of them achieved their baseline eGFR. Previously published works in regard to renal recovery after TDF discontinuation are often retrospective, heterogeneous, and have studied this phenomenon when TDF was stopped because the development of kidney damage $[25,26]$ or irrespectively of the cause of TDF cessation [27]. In these studies most patients experience an improvement in their eGFR (61.4-68.9\%) and recovery of baseline (or predicted) eGFR was common (42-61.4\%). On the other hand, shorter median time of 4 months to achieve eGFR over $60 \mathrm{ml} / \mathrm{min} / 1.73 \mathrm{~m}^{2}$ has been described [26], as compared with 13 months [25] or 18 months in our study. These differences with our findings might be due to the interruption of TDF when other variables were taken into account (e.g. proteinuria, hypophosphatemia) or with different criteria because of the retrospective characteristics of these studies.

Recent IDSA guidelines have been the first to strongly recommend the use of the percentage of eGFR decline from baseline ( $>25 \%)$ with eGFR $\left(<60 \mathrm{ml} / \mathrm{min} / 1.73 \mathrm{~m}^{2}\right)$ to substitute alternative drugs for TDF, although with a low level of evidence [28]. In our study, the best diagnostic performance of CRDRF using eGFR or creatinine was obtained with the percentage of variation of eGFR from baseline (CKD-EPI) at month 30 . While we set a lower value of $-12 \%$ as the optimal cut-off point, this value has to be taken into account as regards an intention to anticipate the CRDRF event.

Phase II and III trials comparing the new formulation tenofovir alafenamide fumarate (TAF) with TDF have found significantly higher declines in the eGFR in the TDF group after short drug exposure periods (median $<70$ weeks) [29-31]. Further data will be needed about the renal safety profile of TAF in the long term.

\section{Conclusions}

Our findings suggest that long-term use of TDF is associated with a bimodal onset (early and late) of a clinically relevant decrease in renal function, which is only partially reversible in the majority of patients. A progressive decline in the eGFR irrespective of the onset of CRDRF was observed. Patients at higher risk to develop CRDRF are those who are older, with arterial hypertension and with lower baseline eGFR.

\section{Conflict of interest}

The authors declared no potential conflicts of interest with respect to the research, authorship, and/or publication of this article. 


\section{References}

1. Wyatt CM, Winston JA, Malvestutto CD, et al. Chronic kidney disease in HIV infection: an urban epidemic. AIDS 2007; 21: 2101-2103.

2. Miller DS. Nucleoside phosphonate interactions with multiple organic anion transporters in renal proximal tubule. J Pharmacol Exp Ther 2001; 299: 567-574.

3. Weiss J, Theile D, Ketabi-Kiyanvash N, et al. Inhibition of MRP1/ ABCC1, MRP2/ABCC2, and MRP3/ABCC3 by nucleoside, nucleotide, and non-nucleoside reverse transcriptase inhibitors. Drug Metab Dispos 2007; 35: 340-344.

4. Cote HC, Magil AB, Harris M, et al. Exploring mitochondrial nephrotoxicity as a potential mechanism of kidney dysfunction among HIV-infected patients on highly active antiretroviral therapy. Antivir Ther 2006; 11: 79-86.

5. Labarga P, Barreiro P, Martin-Carbonero L, et al. Kidney tubular abnormalities in the absence of impaired glomerular function in HIV patients treated with tenofovir. AIDS 2009; 23: 689-696.

6. Cooper RD, Wiebe N, Smith N, et al. Systematic review and metaanalysis: renal safety of tenofovir disoproxil fumarate in HIV-infected patients. Clin Infect Dis 2010; 51: 496-505

7. Campbell TB, Smeaton LM, Kumarasamy N, et al. Efficacy and safety of three antiretroviral regimens for initial treatment of HIV-1: a randomized clinical trial in diverse multinational settings. PLoS Med 2012; 9: e1001290.

8. Arribas JR, Pozniak AL, Gallant JE, et al. Tenofovir disoproxil fumarate, emtricitabine, and efavirenz compared with zidovudine/ lamivudine and efavirenz in treatment-naive patients: 144-week analysis. J Acquir Immune Defic Syndr 2008; 47: 74-78.

9. Gallant JE, Staszewski S, Pozniak AL, et al. Efficacy and safety of tenofovir DF vs stavudine in combination therapy in antiretroviral-naive patients: a 3-year randomized trial. JAMA 2004; 292: 191-201.

10. Ryom L, Mocroft A, Kirk O, et al. Association between antiretroviral exposure and renal impairment among HIV-positive persons with normal baseline renal function: the D:A:D study. J Infect Dis 2013; 207: 1359-1369.

11. Monteagudo-Chu MO, Chang MH, Fung HB, et al. Renal toxicity of long-term therapy with tenofovir in HIV-infected patients. J Pharm Pract 2012; 25: 552-559.

12. Madeddu G, Bonfanti P, De Socio GV, et al. Tenofovir renal safety in HIV-infected patients: results from the SCOLTA Project. Biomed Pharmacother 2008; 62: 6-11.

13. Mocroft A, Lundgren JD, Ross M, et al. Cumulative and current exposure to potentially nephrotoxic antiretrovirals and development of chronic kidney disease in HIV-positive individuals with a normal baseline estimated glomerular filtration rate: a prospective international cohort study. Lancet HIV 2016; 3: e23-32.

14. Zhao Y, Zhang M, Shi CX, et al. Renal Function in Chinese HIV-Positive Individuals following Initiation of Antiretroviral Therapy. PLoS One 2015; 10: e0135462.

15. Nishijima T, Kawasaki Y, Tanaka N, et al. Long-term exposure to tenofovir continuously decrease renal function in HIV-1-infected patients with low body weight: results from 10 years of follow-up. AIDS 2014; 28: 1903-1910.

16. Mulenga L, Musonda P, Mwango A, et al. Effect of baseline renal function on tenofovir-containing antiretroviral therapy outcomes in Zambia. Clin Infect Dis 2014; 58: 1473-1480.

17. Scherzer R, Estrella M, Li Y, et al. Association of tenofovir exposure with kidney disease risk in HIV infection. AIDS 2012; 26: 867-875.

18. Rubio R, Berenguer J, Miro JM, et al. Recommendations of the Spanish AIDS Study Group (GESIDA) and the National Aids Plan (PNS) for antiretroviral treatment in adult patients with human immunodeficiency virus infection in 2002. Enferm Infecc Microbiol Clin 2002; 20: 244-303.

19. Gansevoort RT, Matsushita K, van der Velde M, et al. Lower estimated GFR and higher albuminuria are associated with adverse kidney outcomes. A collaborative meta-analysis of general and high-risk population cohorts. Kidney Int 2011; 80: 93-104.

20. Stevens LA, Schmid CH, Greene T, et al. Comparative performance of the CKD Epidemiology Collaboration (CKD-EPI) and the Modification of Diet in Renal Disease (MDRD) Study equations for estimating GFR levels above $60 \mathrm{~mL} / \mathrm{min} / 1.73 \mathrm{~m}^{2}$. Am J Kidney Dis 2010; 56: 486-495.

21. Gagneux-Brunon A, Delanaye P, Maillard N, et al. Performance of creatinine and cystatin C-based glomerular filtration rate estimating equations in a European HIV-positive cohort. AIDS 2013; 27: 1573-1581.

22. Zimmermann AE, Pizzoferrato T, Bedford J, et al. Tenofovir-associated acute and chronic kidney disease: a case of multiple drug interactions. Clin Infect Dis 2006; 42: 283-290.

23. Pulido F, Fiorante S. Clinical data II. Clinical experience of tenofovir DF in combination with protease inhibitors. Enferm Infecc Microbiol Clin 2008; 26 Suppl 8: 13-18.

24. Walmsley SL, Antela A, Clumeck N, et al. Dolutegravir plus abacavir-lamivudine for the treatment of HIV-1 infection. N Engl J Med 2013; 369: 1807-1818.

25. Wever K, van Agtmael MA, Carr A. Incomplete reversibility of tenofovir-related renal toxicity in HIV-infected men. J Acquir Immune Defic Syndr 2010; 55: 78-81.

26. Bonjoch A, Echeverria P, Perez-Alvarez N, et al. High rate of reversibility of renal damage in a cohort of HIV-infected patients receiving tenofovir-containing antiretroviral therapy. Antiviral Res 2012; 96: 65-69.

27. Jose S, Hamzah L, Campbell LJ, et al. Incomplete reversibility of estimated glomerular filtration rate decline following tenofovir disoproxil fumarate exposure. J Infect Dis 2014; 210: 363-373.

28. Lucas GM, Ross MJ, Stock PG, et al. Clinical practice guideline for the management of chronic kidney disease in patients infected with HIV: 2014 update by the HIV Medicine Association of the Infectious Diseases Society of America. Clin Infect Dis 2014; 59: e96138.

29. Sax PE, Wohl D, Yin MT, et al. Tenofovir alafenamide versus tenofovir disoproxil fumarate, coformulated with elvitegravir, cobicistat, and emtricitabine, for initial treatment of HIV-1 infection: two randomised, double-blind, phase 3, non-inferiority trials. Lancet 2015; 385: 2606-2615.

30. Mills A, Crofoot G Jr, McDonald C, et al. Tenofovir Alafenamide vs. Tenofovir Disoproxil Fumarate in the First Protease Inhibitor-based Single Tablet Regimen for Initial HIV-1 Therapy: A Randomized Phase 2 Study. J Acquir Immune Defic Syndr 2015; 69: 439-445.

31. Sax PE, Zolopa A, Brar I, et al. Tenofovir alafenamide vs. tenofovir disoproxil fumarate in single tablet regimens for initial HIV-1 therapy: a randomized phase 2 study. J Acquir Immune Defic Syndr 2014; 67: 52-58. 